\title{
Multi-chromatic silicon nanocrystals
}

\author{
Benjamin Bruhn ${ }^{1, \star}$, Benjamin JM Brenny ${ }^{2, \star}$, Sidoeri Dekker ${ }^{1}$, Ilker Doğan ${ }^{3,4}$, Peter Schall ${ }^{1}$ \\ and Katerina Dohnalová ${ }^{1}$
}

Silicon nanocrystals (SiNCs) have great potential to become environmental friendly alternatives to heavy-metal containing nanocrystals for applications including medical imaging, lighting and displays. SiNCs exhibit excellent photostability, non-toxicity and abundant resources, but their often reported inefficient and spectrally limited light emission seriously impair their applications. Here we demonstrate a new method that converts SiNCs into an efficient and robust multi-chromatic phosphor. Using $\sim 15$ keV electron-beam irradiation of oxide-capped SiNCs, we introduce several types of color centers into the nanocrystal's oxide shell with efficient blue, green and red emission bands, together yielding warm-white photoluminescence, even for a single SiNC. Introduced centers are not native to the original system and we relate them to known defects in silica. Unlike in the silica host, however, here the centers are efficiently optically excitable. Provided further optimization and up-scaling of this method, e-beam irradiated SiNCs can be of great interest as white phosphors for applications such as LEDs.

Light: Science \& Applications (2017) 6, e17007; doi:10.1038/lsa.2017.7; published online 30 June 2017

Keywords: cathodoluminscence; color center; electron beam; multi-chromaticity; silicon nanocrystals

\section{INTRODUCTION}

In the quest for ever more energy-efficient, cost-effective and colorenhancing light emitters for display technologies and light-emitting diodes (LEDs), much research has focused on semiconductor nanocrystals (NCs). They generally offer bright emission with high quantum yield that is tunable throughout a wide color range by simple variation of their size, which sets their energy level structure. While some materials such as cadmium selenide present a health risk and are subject to legislative bans for the consumer electronics market in Europe, nanostructured silicon is seen as a promising alternative due to its photostability, non-toxicity, biodegradability and abundance of resources ${ }^{1}$. Nevertheless, application of silicon NCs (SiNCs) in commercial technologies is currently hindered by the fact that their properties vary markedly with surface capping, emission yields are low and fully balanced emission spectra in the visible are very difficult to obtain $^{1}$. Here we focus on possible broadening of the limited spectral properties.

While hydrogen-capped silicon nanocrystals (H-SiNCs) do exhibit some spectral tunability of photoluminescence $(\mathrm{PL})^{1-3}$, they are prone to oxidation ${ }^{1,4,5}$. The resulting oxide-capped SiNCs (O-SiNCs) show size-tunable emission only for larger NCs emitting in the near-IR range ${ }^{1-5}$. With decreasing size, their emission becomes dominated by surface-related species, switching from a broad red PL band at around $2 \mathrm{eV}(620 \mathrm{~nm})^{4-8}$ to a broad blue band at around $2.8 \mathrm{eV}(450 \mathrm{~nm})^{1,6,9-15}$, skipping the green spectral region completely ${ }^{6}$. A third common type, oxide-free organically capped SiNCs (C-SiNCs), does show direct band gap-like emission properties $^{16-18}$, but is difficult to prepare at larger sizes that would emit in the green-red spectral ranges ${ }^{1}$. Hence, there is urgent need for an alternative method to achieve bright, white-light-emitting SiNCs for lighting applications.

In bulk and nanostructured silica $\left(\mathrm{SiO}_{2}\right)$, spectrally rich absorption and emission can be achieved through defects (color centers), created under strain, irradiation or at interfaces (for example, Refs. 19-39). In stoichiometric bulk $\mathrm{SiO}_{2}$, each $\mathrm{Si}$ atom binds covalently to four $\mathrm{O}$ atoms and each $\mathrm{O}$ atom to two $\mathrm{Si}$ atoms, with flexible angular conformation and high binding energy $(4.5 \mathrm{eV})^{20}$. When these binding configurations change, defects are created that can provide efficient luminescent recombination pathways with characteristic emission spectra. Common types of defects contribute in the visible spectral range with bright blue, green and red bands. Several of these are depicted in Figure 1a (for a more complete overview of silica defects active as color centers, see Supplementary Information and Refs. 19-39). Unfortunately, in silica, these color centers can only be efficiently excited by high-energy photons or an electron beam due to the large band gap of the silica host, precluding their use in lighting applications.

Here we show, for the first time, that similar color centers can be used to broaden the emission spectra of SiNCs with originally much narrower and quite limited emission spectral range, and this happens independently of the original SiNCs emission spectral

\footnotetext{
${ }^{1}$ Van der Waals-Zeeman Institute, University of Amsterdam, Science Park 904, Amsterdam 1098 XH, The Netherlands; ${ }^{2}$ Center for Nanophotonics, AMOLF, Science Park 104, Amsterdam 1098 XG, The Netherlands; ${ }^{3}$ Department of Applied Physics, Eindhoven University of Technology, P.0. Box 512, Eindhoven 5600 MB, The Netherlands and ${ }^{4}$ Dutch Institute for Fundamental Energy Research (DIFFER), P.O. Box 6336, Eindhoven 5600 HH, The Netherlands

*These authors contributed equally to this work.

Correspondence: K Dohnalová, Email: k.dohnalova@uva.nl

Received 1 August 2016; revised 1 February 2017; accepted 8 February 2017; accepted article preview online 10 February 2017
} 
a

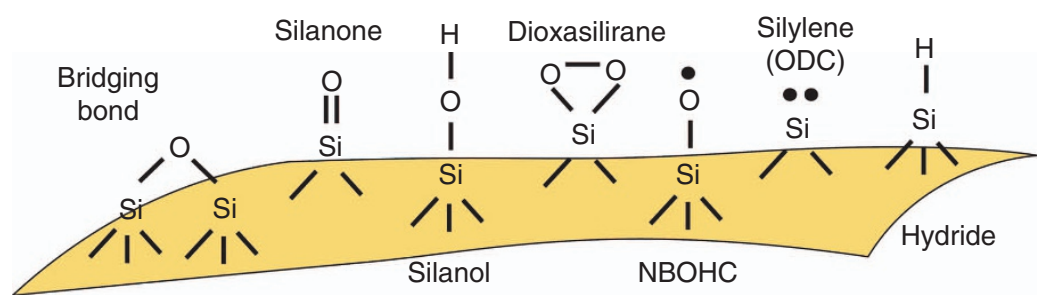

b
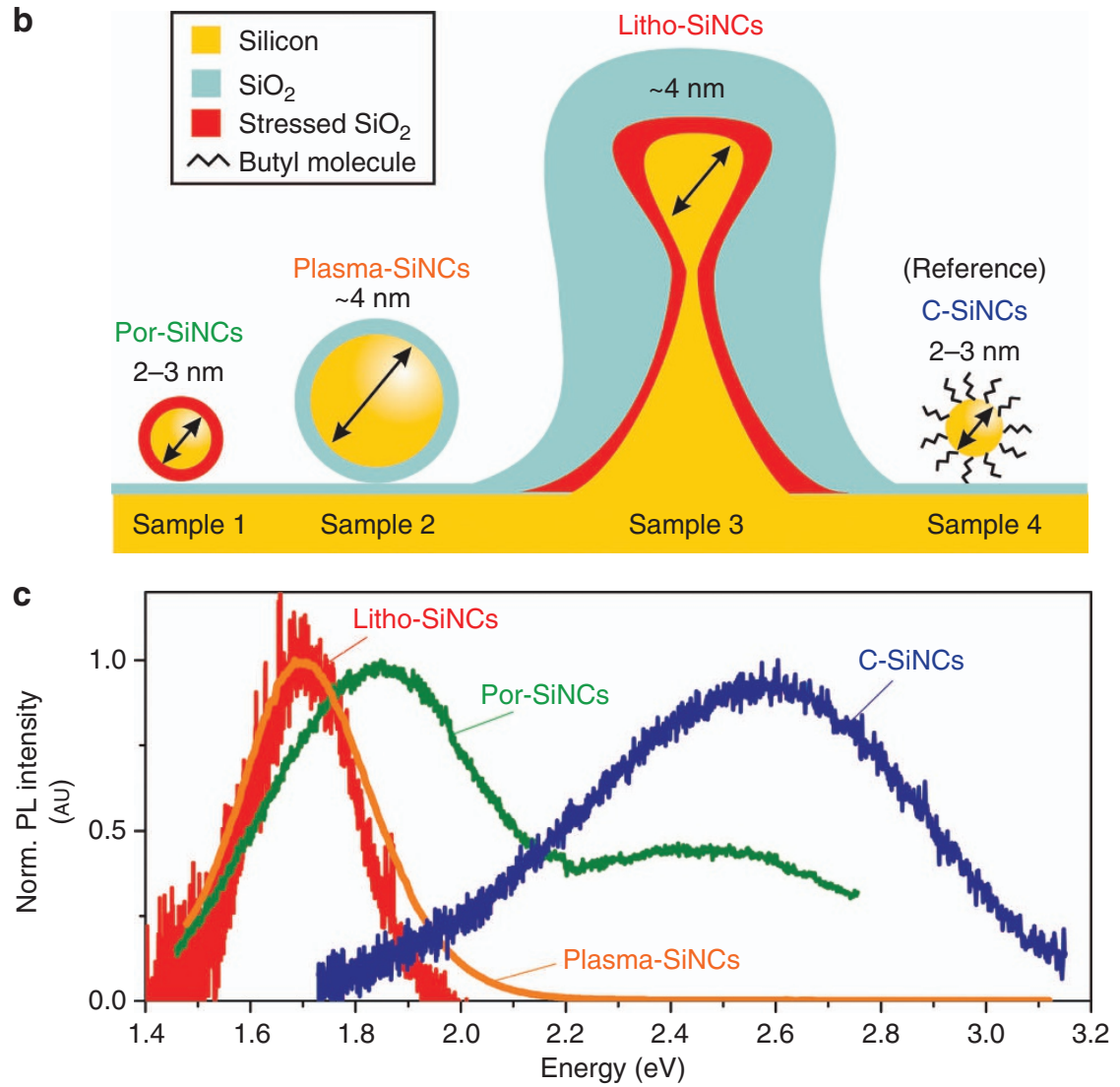

Figure 1 Color centers and SiNCs samples. (a) Color centers observed in bulk and nanostructured silica: (from left to right) Bridging $\equiv \mathrm{Si}-\mathrm{O}-\mathrm{Si} \equiv$ bond ${ }^{7,8}$, silanone $=\mathrm{Si}=\mathrm{O}(\operatorname{Refs} 4,8,19)$, silanol $\equiv \mathrm{Si}-\mathrm{OH}^{28,29}$ and its derivatives dioxasilirane $=\mathrm{Si}\left(\mathrm{O}_{2}\right)^{22}$, silylene $=\mathrm{Si} \bullet \bullet($ Refs $20,21,24,35$; also known in bulk silica as oxygen-deficiency center, ODC ${ }^{20,21}$ ), non-bridging oxygen hole center (NBOHC) $\equiv \mathrm{Si}-\mathrm{O} \bullet$ (Refs 20,21,30-34) and Si-hydride $\equiv \mathrm{Si}$ - $\mathrm{H}^{27}$ (see Supplementary Information for more details). (b) Sketch of the four types of SiNCs studied here; samples differ in size, surface capping and oxide strain. (c) Normalized PL from the four types of SiNCs samples before e-beam irradiation. Intensities are not to be directly compared due to different emission efficiencies and SiNCs concentrations in the studied samples. The oxide-free C-SiNCs exhibit strong emission at around $2.6 \mathrm{eV}$ due to band-to-band excitonic recombination ${ }^{17,40}$. The Por-SiNCs shows two equally strong oxide-related bands at $2.5 \mathrm{eV}$ and $1.8 \mathrm{eV}$ related to the silica oxide capping ${ }^{6}$. Plasma-SiNCs ${ }^{41}$ and Litho-SiNCs ${ }^{42}$ are both larger in core size and both show intrinsic PL around $1.7 \mathrm{eV}$. For litho-SiNCs the PL signal is weak, because it is measured from a single SiNC emitter.

shape. The color centers are introduced into the oxide shell of O-SiNCs by mild $(2-30 \mathrm{keV})$ e-beam irradiation, where they become optically excitable in blue spectral range, due to the small band gap of the SiNC core. These color centers lead to new emission bands that are not naturally occurring in O-SiNCs and are stable in air. These emission bands, particularly including the missing green band ${ }^{6}$, have similar brightness throughout the whole visible range, turning the treated SiNCs into a well-equilibrated multi-chromatic emitter that luminesces with a warmly white color. This is promising for multiple applications, but much larger quantities of material will be needed for a detailed analysis of figures of merit of the newly acquired emission properties. Nevertheless, our observations show that this material is of great interest for the white phosphor LED industry.

\section{MATERIALS AND METHODS}

To offer comprehensive comparison of various types of SiNCs, four different materials were studied. Three types of oxide capped SiNCs (O-SiNCs) were used, differing in size, preparation protocol and oxide-capping thickness and quality, and as a reference we studied oxide-free sample of organically capped nanocrystals (C-SiNC). For sample overview, see Table 1 and sketch in Figure $1 \mathrm{~b}$.

First sample, Por-SiNC (Sample 1) consists of small NCs with diameter of $2-3 \mathrm{~nm}$ that are made from mechanically pulverized layers of oxidized porous silicon that has been additionally post-etched in hydrogen peroxide solution, leading to increased amounts of $-\mathrm{OH}$ surface moieties (see Dohnalová et al. ${ }^{6}$ for more information). Second sample, Plasma-SiNCs (Sample 2), contains larger NCs with core diameter of around $4 \mathrm{~nm}$, prepared by plasma synthesis from silane 
Table 1 Four types of studied SiNCs samples

\begin{tabular}{|c|c|c|c|c|c|}
\hline Sample & Name & Capping & Diameter [Reference] & Distribution & Preparation [Reference] \\
\hline 1 & Por-SiNCs & Naturally grown thin silica oxide & $2-3 n^{6}$ & Ensemble & Electrochemical etching 6 \\
\hline 2 & Plasma-SiNCs & Naturally grown thin silica oxide & $\sim 4 \mathrm{~nm}^{41}$ & Ensemble & Plasma synthesis 41 \\
\hline 3 & Litho-SiNCs & Strained thick silica oxide & $\sim 4 \mathrm{~nm}^{42}$ & Single NC & Electron beam lithography 42 \\
\hline 4 & C-SiNCs & Organic (alkyl) & $\sim 2.2 \pm 0.5 \mathrm{~nm}^{17,40}$ & Ensemble & Wet-chemical synthesis ${ }^{17,40}$ \\
\hline
\end{tabular}

Additional material and spectroscopic properties of these samples have been extensively studied and reported elsewhere. More details are given in the Experimental section, Supplementary Information (Supplementary Fig. S1) and respective Refs 6,17,40-42.

gas. The NC naturally oxidized by exposure to air, which results in a thin, non-thermal oxide layer (more information can be found in Doğan et al. ${ }^{41}$ ). Finally, third sample, Litho-SiNCs (Sample 3), contains larger Si nanostructures, some of them similarly sized as those of the Plasma-SiNC sample, which we chose for this study. This sample was prepared by electron-beam lithography followed by reactive ion-etching and self-limiting oxidation (more details can be found in Bruhn et al. ${ }^{42}$ ). This oxidation technique results in SiNCs embedded in a thick strained thermal oxide as indicated in the sketch in Figure 1b. The reference sample C-SiNCs (Sample 4) was prepared by wet-chemical synthesis and have small core diameter of $(2.2 \pm 0.5) \mathrm{nm}$ and surface passivated by butyl ligands (more details can be found in Refs. 17,40).

For the microscopic analysis, all colloidal nanocrystals (Por-, Plasma- and C-SiNC samples) were dispersed and sonicated in UVgrade ethanol and drop-casted onto a clean pre-patterned silicon surface. The patterned substrate allowed us to recognize and analyze the same microscopic sample area in the PL and CL measurements. Silicon substrates are covered with a naturally grown thin oxide layer that exhibits negligible PL and CL signals (see Supplementary Information, Supplementary Figs. S5a and S5b), but is sufficiently conductive. For better contrast, the weakly emitting Plasma-SiNCs were deposited onto a glass substrate partially covered by a thin gold sheet. We confirmed that this change of substrates had no influence on the emission spectrum. For more detailed information and analysis of the samples, see Supplementary Fig. S1 and respective Refs. 6,40-42.

For spectral analysis, we used photoluminescence (PL) and cathodoluminescence (CL) micro-spectroscopy setups. Steady-state microPL was measured using an inverted wide-field optical microscope (Zeiss AxioVert XY, Zeiss, Oberkochen, Germany) with $\times 100$ air objective (NA 0.7) as shown in Figure 2a. The PL was excited by a $\sim 3.1 \mathrm{eV}(405 \mathrm{~nm})$ low power $(<5 \mathrm{~mW}) \mathrm{cw}$ laser diode and detected by a liquid-nitrogen-cooled CCD camera (Pylon 400B, Princeton Instruments, Acton, MA, USA) coupled to a spectrometer (Acton SP2300, Princeton Instruments). PL spectra were measured at room temperature and ambient conditions and corrected for background and spectral response of the detection system. The CL measurements were performed in the vacuum chamber of the scanning electron microscope (SEM; FEI XL-30 SFEG) $)^{43,44}$ as shown in Figure $2 b$. Samples were irradiated by a focused e-beam of diameter of $\sim 2-$ $10 \mathrm{~nm}$, electron energies in range of $2-30 \mathrm{keV}$ and beam currents of $0.2-2 \mathrm{nA}$, to generate color centers and excite the CL emission. In each step, CL is collected from area of approximately $100-200 \mathrm{~nm}^{2}$ and the beam scanning mode covered an area of approximately $10 \mu \mathrm{m} \times 10 \mu \mathrm{m}$. This area was large enough to get measurable signal in the follow-up micro-PL measurements to conclude spectral changes after the irradiation. The CL signal is collected by a parabolic mirror and deflected into a liquid-nitrogen-cooled front-illuminated Si CCD camera (Spec-10 100F, Princeton Instruments), coupled to a spectrometer (Acton SP2300, Princeton Instruments). CL has been measured under various exposure times and varying electron energies at room temperature and has been corrected for the spectral response of the detection system.

\section{RESULTS AND DISCUSSION}

To investigate the generation of color centers in the oxide shell of the O-SiNCs, we studied micro-PL and micro-CL emission spectra before, during and after e-beam irradiation. Three different types of O-SiNCs were studied (Figure $1 \mathrm{~b}$ and Table 1), together with the reference oxide-free C-SiNCs (Supplementary Fig. S4). Samples differ in the NC core size, surface capping and oxide shell strain, allowing us to elucidate the origin and robustness of the induced spectral changes. Essentially, samples 1 and 4 have similar core size, but different capping (oxide vs. organic). Samples 1 and 2 have oxide capping, but different size $(\sim 2.5 \mathrm{~nm}$ vs. $\sim 4 \mathrm{~nm})$. Samples 2 and 3 have similar sizes, but their silica shells differ in thickness, strain and interface quality (natural thin oxide vs. strained thick thermal oxide). Another difference concerns the measurements where those on samples 1,2 and 4 were measured on larger numbers of SiNCs at once ('ensemble'), those on sample 3 represent single-nanoparticle measurements due to the extremely low concentration of SiNCs in this sample. These largely different samples allow us to elucidate the generic effect of e-beam irradiation on the emission spectra of the SiNCs and their relation to the oxide shell of $\mathrm{O}-\mathrm{SiNC}$.

To study the induced emission changes by the e-beam treatment, we compare the steady-state micro-PL of the O-SiNCs before and after the irradiation (Figure $2 \mathrm{c}$ and $2 \mathrm{~d}$, respectively), measured under ambient conditions using inverted wide-field optical microscope coupled to a spectrometer (Figure 2a). To generate color centers, samples were irradiated with a focused e-beam in a SEM (Figure 2b). E-beam irradiation also excites the emission in the form of cathodoluminescence (CL), which is analyzed in situ by the integrated spectroscopic CL system. To monitor the spectral changes during the irradiation, we alternated short and long e-beam exposures (Figure $2 \mathrm{e}$ and $2 \mathrm{f}$, respectively): the short ( $\sim \mathrm{few}$ seconds) exposures leaving emission spectra unchanged, the long ( $\sim$ few minutes) exposures introduce defects that act as color centers, continuously changing the emission spectra. After the treatment, samples were removed from the SEM vacuum chamber, and their micro-PL was studied again.

The micro-PL spectra before e-beam irradiation are show in Figure $2 \mathrm{c}$ and reveal the expected spectral properties: absence of green $^{6}$ (Por-SiNCs) and the complete absence of green-blue emission (Plasma-SiNCs and Litho-SiNCs). The apparent differences in the spectra reflect the different core size and surface capping of the SiNCs associated with their different preparation. Strikingly, after the e-beam irradiation, very different micro-PL spectra, with much enhanced green-blue emission, are observed for all O-SiNCs (Figure 2d), measured from the same areas as before irradiation. Irrespective of 

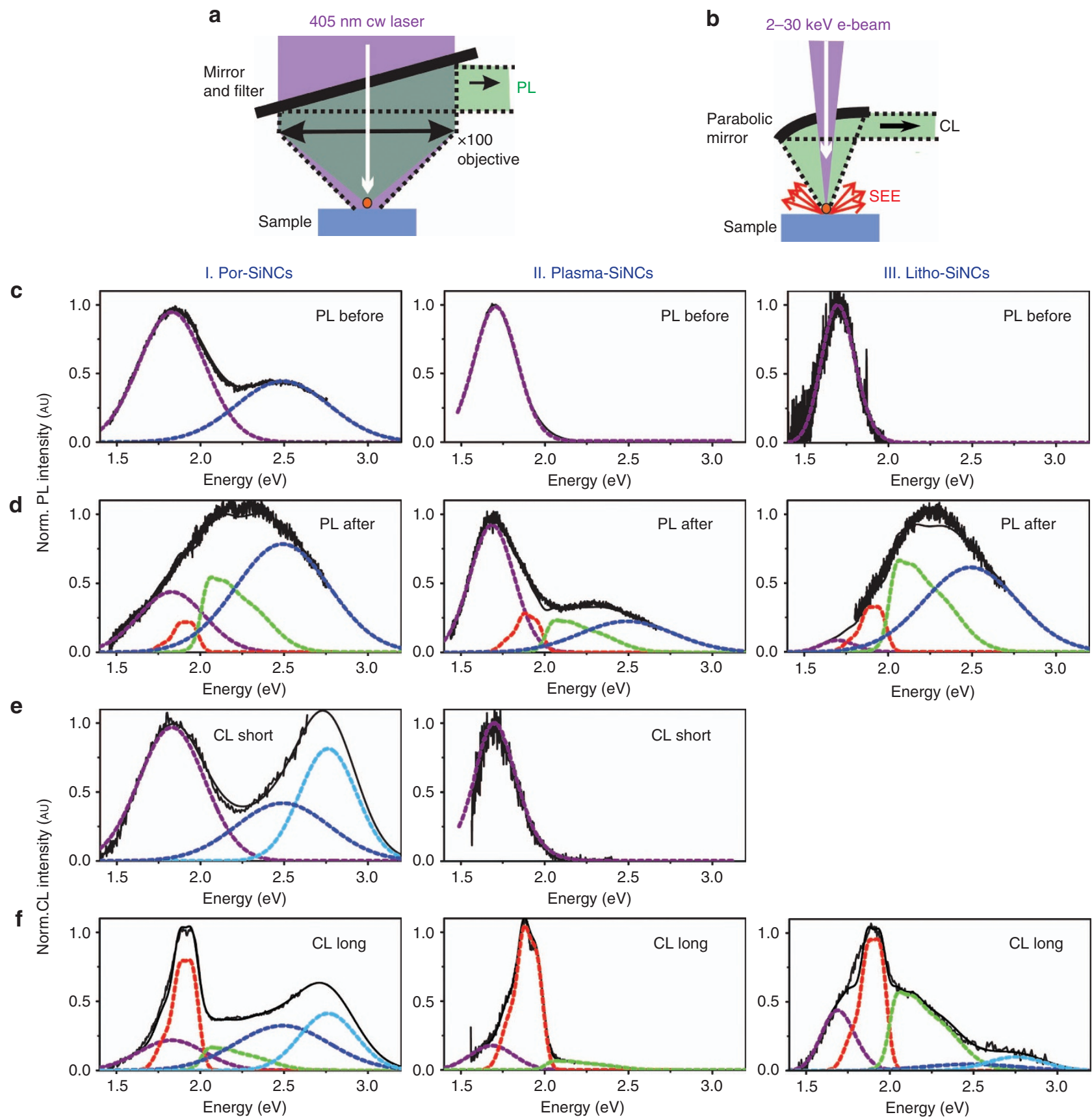

Figure 2 Spectral changes in O-SiNCs upon irradiation. Sketch of the (a) PL and (b) CL microscopy setup: For the PL, we used a $\sim 3.1 \mathrm{eV}$ (405 $\mathrm{nm}$ ) continuous wave laser diode for excitation and a microscope with a coupled spectrometer and CCD camera for detection. For CL, excitation by an e-beam with energy variable between $2-30 \mathrm{keV}$ passes through a small opening in a parabolic mirror that is used to collect $\mathrm{CL}$ emission and direct it towards a spectrometer coupled to a CCD. (c,d) PL emission spectra of all O-SiNCs taken before and after e-beam irradiation. (e,f) CL spectra taken for short and long e-beam exposure. For CL measurements here we used $15 \mathrm{keV}$ electron energy, which is close to the optimal excitation energy (see Supplementary Fig. S4b for Por-SiNCs). Colored lines represent fits of the contributing bands: the original PL bands (dark blue and violet lines) and the created color centers emitting in red, green and blue spectral ranges (light red, green and light blue lines, respectively). We note that the short exposure CL data were not possible to retrieve for the Litho-SiNC, due to the single nanocrystal nature of the measurement. All the PL and CL spectra are corrected for the spectral sensitivity of the detection system.

their different initial spectra, all samples feature, in addition to their original PL bands, a new broad PL band that can be fitted with several new emission bands: a bright blue band at around $2.5 \mathrm{eV}$; a bright green band around $2.2 \mathrm{eV}$; and a narrow red band at $1.9 \mathrm{eV}$, broadening the spectrum towards the visible. The choice of the fitting parameters will be explained later.
To investigate the spectral changes in more detail, we look at the development of the CL spectra during the e-beam irradiation. The short exposures were short enough not to generate new defects and only probe the emission spectra of the samples under the higher energy excitation $(15 \mathrm{keV})$, as shown in Figure 2e. Red/NIR emission bands remained unchanged, and only the blue band in Por-SiNCs is 


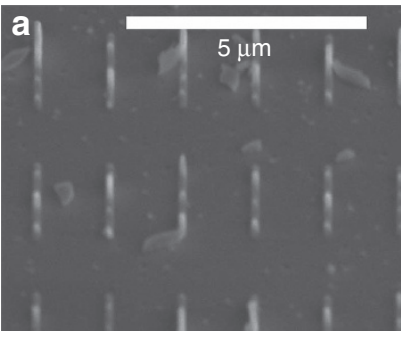

b

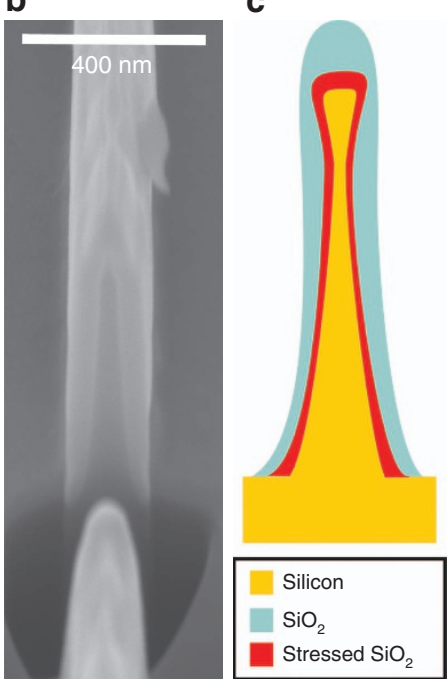

d

Spot 3

Spot 2

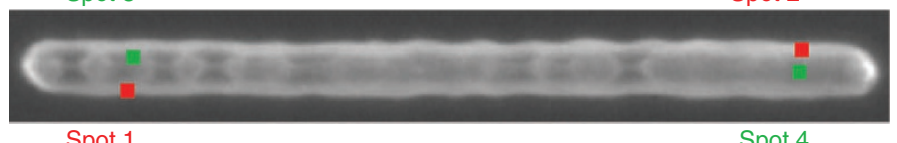

Spot 1

Spot 4

$\mathbf{e}_{(1-4)}$
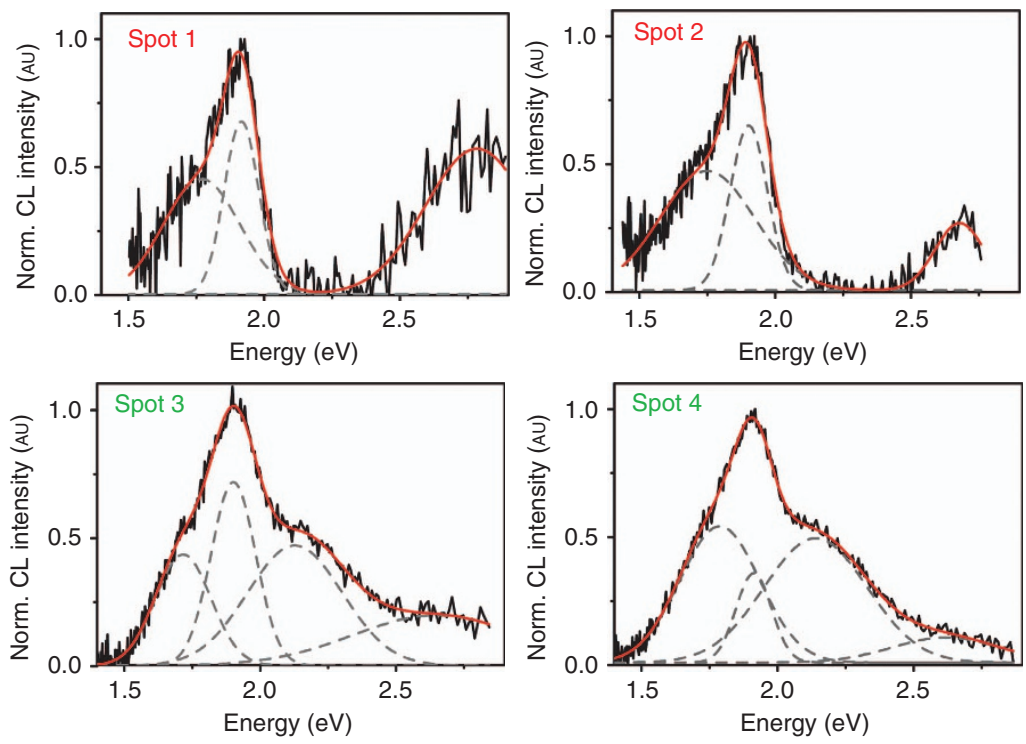

Figure 3 Spatially resolved study of Litho-SiNC sample. SEM image of several Si nanowalls (a) and a single nanowall cut through by ion beam (b), revealing the Si core and silica wall sides. (c) Sketch of the cross-section of the nanowall. (d) SEM image of the nanowall from the top with approximately indicated positions of the measurements that coincided with the presence of a SiNC. (e) CL spectra from the side of the nanowall (spot 1 and 2 ) and from the center (spot 3 and 4), just above the SiNC.

blue shifted, with respect to original PL. To emphasize that, we added extra blue band at $2.76 \mathrm{eV}$. In contrast, the long exposures lead to appearance of new emission bands, eventually resulting in very different spectral characteristics, as shown in Figure 2f. Already after a few minutes of e-beam exposure, the CL spectra show new emission bands-a narrow red band appears at around $1.9 \mathrm{eV}$, a green emission band at around $2.2 \mathrm{eV}$, and a blue band appears at around 2.7-2.8 eV nm (except for the Plasma-SiNCs). The gradual rise of the new bands upon the irradiation is clearly seen in the time evolution of the CL intensity in Supplementary Information, Supplementary Figs. S5 and S6. Where Por-SiNC and Plasma-SiNCs have only very thin silica shells, Litho-SiNC has very thick silica shell and hence spatially resolved CL study was possible, shown in Figure 3. This sample consists of thermally oxidized Si nanowalls (Figure 3a-3d) until the point when the utmost top part of the Si nanowall separates and forms nanocrystal. Near-infrared PL emission around $1.7 \mathrm{eV}$ (Figure 1c) occurs from places in the nanowall where the nanocrystal is formed. Under e-beam irradiation, we found that CL from the sides of the nanowall (Figure 3e, spot 1 and 2) differs from the CL from the center of the nanowall (Figure $3 \mathrm{e}$, spot 3 and 4), i.e. just above the SiNC. In stark contrast to the all O-SiNCs, the reference oxide-free C-SiNCs sample does not show any change upon irradiation, as can be seen in Supplementary Figs. S4a and S4c. Furthermore, signal contribution from the reference silicon substrate with a native silica layer is negligible (Supplementary Fig. S5b). Hence, we conclude that the new spectral features are related to the presence of the silica oxide shell on the O-SiNCs.
To follow the spectral evolution of the emission bands quantitatively, we fit all the PL and CL spectra shown here with a fixed set of peaks with fixed full-width of half maximum and fixed peak position, adjusting only their relative amplitudes between various samples and experimental stages. The peak parameters are retrieved from the best available measurements: for the blue, green and red bands (light blue, green and red lines) we used the time-scans and spatially resolved measurements in the Supplementary Figs. S5 and S6 and Figure 3e, and for the original PL bands (dark blue and violet lines) we used the data of Figure $2 c$. As shown in Figure $2 c-2 f$, we can describe all the observed PL and CL spectra of any of the three O-SiNCs samples from the superposition of just five major spectral contributions.

The robustness of these spectral features indicates constant underlying mechanisms. In fact, a close look at the characteristic spectral profile shows that we can relate all the emerging spectral features to well-known color centers in bulk and nanostructured silica ${ }^{19-39}$ : The very bright narrow red $\sim 1.9 \mathrm{eV}$ band that contributes significantly in all samples is likely related to the silica $\mathrm{NBOHC}$ surface variant color center (Figure 1a), as is evident from its characteristic doublepeak feature $e^{30,31}$. In the final PL spectra, this band is less intense, which is expected due to its reduced excitation cross-section at $3 \mathrm{eV}^{45}$. The green CL band around $2.2 \mathrm{eV}$ is the most interesting as to the best of our knowledge it does not occur in the native form of the oxide shell of O-SiNCs ${ }^{1}$. From our observation in Figure 3e, we conclude that an Si-rich environment is essential, as well as the presence of strain $^{38}$, as the green band consistently shows up only at the Si-rich center of the Si nanowall, but not at all at the silica-rich sides of the wall. Also, it is the brightest in the litho-SiNCs that exhibit the thickest 


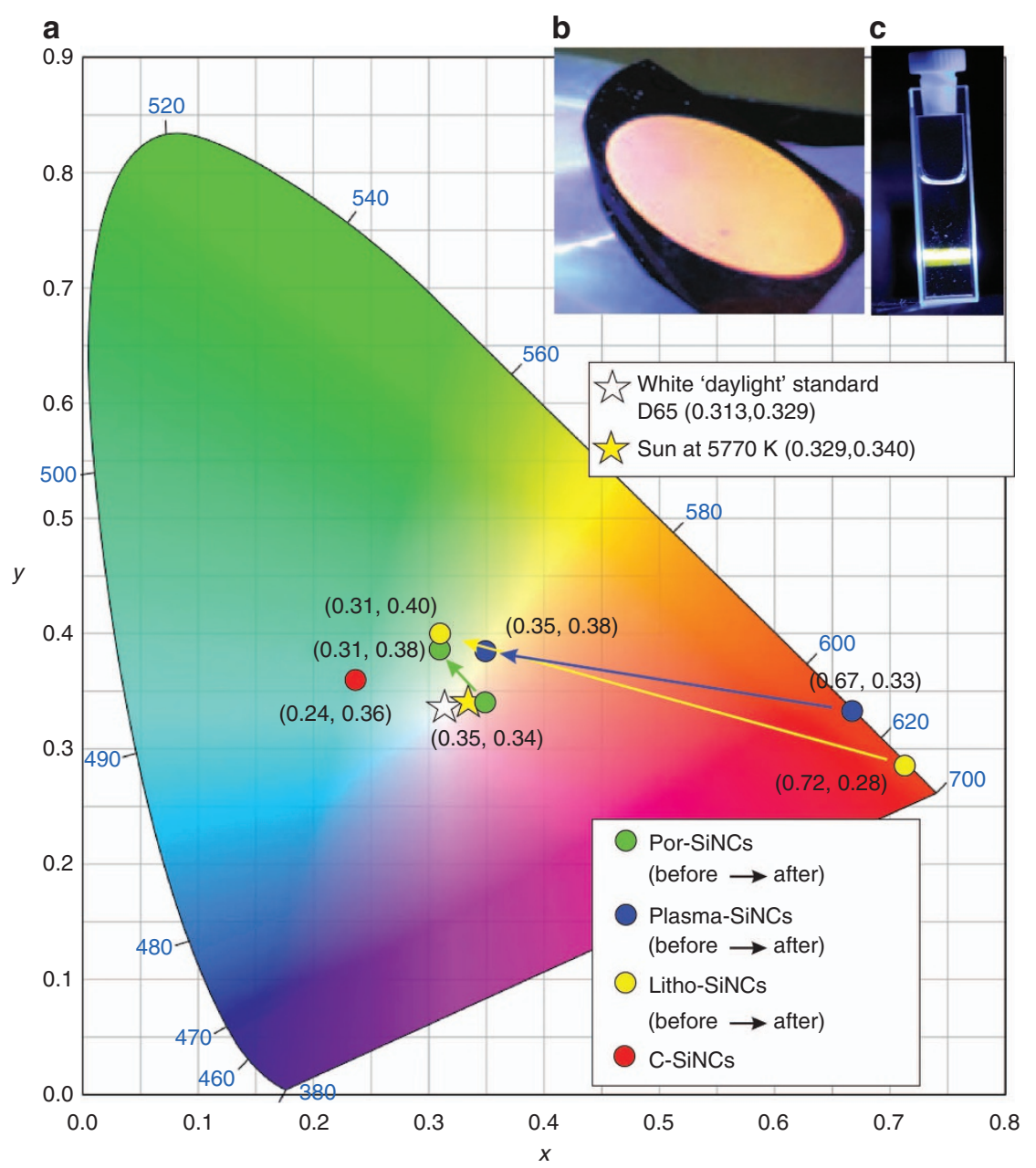

Figure 4 Resulting color changes. (a) Color gamut for PL spectra of all the studied SiNC samples before and after extended e-beam irradiation. CIE coordinates are shown for each sample in the graph and arrows indicate the shift for each sample induced by the e-beam treatment (For the C-SiNCs the position does not change, because irradiation did not lead to any spectral modifications). For comparison, we show also white standard D65 and the Sun's spectrum position. For the color gamut evaluation, CIE 1931 standards were used. Insets: Real-color photos of native Por-SiNC and C-SiNC, both exhibiting whitish color PL that is improved in por-SiNCs by introduction of the green center.

and the most strained silica shell of all the studied samples. It might be possible that green color centers like dioxasilyrane $=\mathrm{Si}\left(\mathrm{O}_{2}\right)^{23}$ or $\equiv \mathrm{Si}-$ $\mathrm{H}^{27}$ and $=\mathrm{Si}=\mathrm{O}^{4}$ (the latter two reported unstable on the surface of the nanostructured silica ${ }^{19}$ ), are stabilized here within the oxide shell of the SiNCs. Finally, the blue emission band showing in CL at 2.7$2.8 \mathrm{eV}$ and in PL at $2.5 \mathrm{eV}$ is possibly of the same origin and might originate from the oxygen-deficiency centers (silylene in Figure 1a) ${ }^{20,35}$ and/or the defect pair consisting of a dioxasilyrane, $=\mathrm{Si}\left(\mathrm{O}_{2}\right)$, and a silylene $=\mathrm{Si} \cdot{ }^{37}$. To be able to distinguish between the two, more detailed time-resolved PL and CL analysis is needed. Nevertheless, from our spectral analysis it appears likely that both blue bands observed are of the same origin and occur from oxide centers already existing before irradiation, as it appears to be already present in one of the as-grown samples (por-SiNCs). This is supported by the fact that oxygen-deficiency centers can appear as a result of reactions between neighboring silanol $\equiv \mathrm{Si}-\mathrm{OH}$ groups following the reaction scheme $\left[\equiv \mathrm{Si}-\mathrm{O}-\mathrm{H}+\mathrm{H}-\mathrm{O}-\mathrm{Si} \equiv \leftrightarrow \mathrm{ODC}+\mathrm{H}_{2}\right]$ and $\mathrm{Si}-\mathrm{O}-\mathrm{H}$ groups are abundant on the surface of our Por-SiNC sample due to a specific post-etch procedure in hydrogen peroxide ${ }^{6}$. By comparing the original PL with the short-exposure CL spectra (por-SiNC in Figure 2e and 2f), we conclude that no new blue-emitting centers are formed. The slight blue-shift of the blue band in the CL compared to the PL can be caused by the different excitation energies, as the blue PL band has been reported to shift under different optical excitation energies ${ }^{13}$. Interpretation of the blue bands as native to O-SiNC shell is consistent with measurements of Plasma-SiNC PL shown in Supplementary Fig. S8, where strong blue PL band develops purely by long term $(\sim 1$ year) exposure to air.

Considering spectral similarities, time-scans and spatial-analysis of the newly formed emission bands, we suggest that color centers similar to those in silica materials are formed here in the silica-oxide shell of the O-SiNCs. However, unlike in silica, their PL emission can be efficiently excited optically at $\sim 3.1 \mathrm{eV}(405 \mathrm{~nm})$ using low power $(<5 \mathrm{~mW})$ laser diode, due to the comparably much narrower band gap of our nanocrystals $(\sim 1.7-1.8 \mathrm{eV})$. Besides inherent non-toxicity and abundancy of silicon resources, this is yet another major advantage these materials, opening possibility of optically pumped white phosphors for LEDs.

Spectral changes introduced by the e-beam treatment can be summarized in the color gamut chart in Figure 4a. As a result of the irradiation, all O-SiNCs change their spectral coordinates to very similar end-chromatic coordinates associated with the introduced red, 
green and blue color centers. The resulting PL emission of all irradiated samples appears warmly white to the eye, with the warmest white obtained for the originally near-infrared emitting Plasma-SiNC. This is different from the reference oxide-free C-SiNC, whose chromatic coordinates do not change by the irradiation, remaining cold blue-white (Figure 4a, red dot and photo in inset Figure 4c). The Por-SiNC sample, as discussed before, shows whitish PL already in its native state (Figure 4a, green dot and inset Figure 4b), but also here the introduction of the missing green emission band results in a better defined white color. The most interesting and pronounced spectral changes from far-red to white color, however, occur for the Plasmaand Litho-SiNC samples. For the latter, the single-nanocrystal measurements indicate that the broad emission spectrum is indeed a single-nanocrystal property, and not an ensemble effect. Importantly, this identifies even a single nanocrystal as a multi-chromatic luminescent emitter with white spectral profile.

Intensity of the induced color center's PL appears to be stable in air for all the samples for several days, which was the typical delay between CL irradiation and subsequent micro-PL measurements. While the spectral shape of emission always changes drastically during e-beam irradiation, the overall integral CL intensity seems to be either stable (Plasma-SiNCs, see Supplementary Figs. S5c and S5d) or slightly enhanced by a factor 2-3 (Por-SiNCs, Supplementary Fig. S6). High resolution of the CL micro-spectroscopy, however, makes is hard to give a definite enhancement factor, due to inhomogeneity of the dropcasted materials in nano- and micro-scales (at every point, signal is collected by focused e-beam of $2-10 \mathrm{~nm}$ diameter, from diffraction limited area of $100-200 \mathrm{~nm}^{2}$ ). For micro-PL, we found that similar behavior occurs-PL intensity appears to be either similar (PlasmaSiNC) or slightly enhanced by factor $\sim 2$ (Supplementary Figs. S7a and S7b). Again, high spatial resolution and nano-/micro-scale inhomogeneity of the drop-casted SiNC samples makes it difficult to give final enhancement factor. Importantly, we have to keep in mind in such evaluations that excitation cross-section for each color center and SiNC core are very different.

\section{CONCLUSIONS}

Environmental friendly SiNCs with a spectrally limited emission profile can be turned into a phosphor with a well-balanced visible white emission spectrum upon e-beam irradiation. The spectral changes induced by the e-beam irradiation consist of a considerable spectral shift as well as broadening, irrespective of the initial sample characteristics. We conclude that the irradiation creates new color centers in the oxide shell of the O-SiNC, since the oxide-free organically capped C-SiNCs do not show any change in their emission spectrum upon irradiation and emission from defect centers generated in the native oxide thin film on the Si substrate is negligible. These defects can be related to well-known color centers in bulk and nanostructured silica. Unlike in silica, however, these color sites can be efficiently optically excited in the SiNC systems at $405 \mathrm{~nm}$ by a low power laser diode, due to the narrower band gap of the host. From the comparison of the three very different O-SiNC samples, we found this effect to be independent of the preparation protocol and properties of each particular type of O-SiNC, with small variations: whereas the green band emission benefited from thicker and possibly more strained silica shells, red and blue bands appeared in all samples, with the blue band being exceptionally well-developed and stable in air. We thus believe that any oxide-capped SiNC, treated by an e-beam with an energy of around $15 \mathrm{keV}$ for a few minutes can be converted into a brightly warm-white light-emitting material, especially provided a thicker silica shell. This constitutes a novel, robust, and reliable method to convert any environmental friendly and abundant (oxidecapped) SiNCs into a white color phosphor. Further optimization of the material and emission yields is necessary, however, the current study shows that this effect offers many properties sought by whitelight applications.

\section{CONFLICT OF INTEREST}

The authors declare no conflict of interest.

\section{ACKNOWLEDGEMENTS}

We acknowledge Professor Jan Linnros for providing Litho-SiNCs sample, Professor H. Zuilhof for providing the C-SiNCs sample, Professor M. C. M. van de Sanden for providing the Plasma-SiNCs and Professor A. Polman for fruitful discussions and enabling the CL measurements. This work was supported by a MacGillavry Fellowship (K.D.), the Stichting voor Fundamenteel Onderzoek der Materie (FOM), which is part of the Netherlands Organisation for Scientific Research (NWO; K.D., I. D.), NanoNextNL program (I.D.) funded by the Dutch Ministry of Economic Affairs and Vici fellowship from NWO (B.B. and P.S.). Author contributions are as follows: K.D. initiated and supervised the project, Bbruhn, Bbrenny, S.D. and K.D. performed the measurements, data analysis and data interpretation. Bbruhn, Bbrenny, K. D. and P.S. co-wrote the paper. All authors discussed the results and contributed to the interpretation of the data.

1 Dohnalová K, Gregorkiewicz T, Kůsová K. Silicon quantum dots: surface matters. J Phys Condens Matter 2014; 26: 173201.

2 Hybertsen MS. Absorption and emission of light in nanoscale silicon structures. Phys Rev Lett 1994; 72: 1514-1517.

3 Delerue C, Allan G, Lannoo M. Theory of silicon nanocrystals. In: Pavesi L, Gaponenko $S$, Dal Negro L editors. Towards the First Silicon Laser. The Netherlands: Springer. 2003, pp 243-260.

4 Wolkin MV, Jorne J, Fauchet PM, Allan G, Delerue C. Electronic states and luminescence in porous silicon quantum dots: the role of oxygen. Phys Rev Lett 1999; 82: 197-200.

5 Dohnalová K, Kůsová K, Pelant I. Time-resolved photoluminescence spectroscopy of the initial oxidation stage of small silicon nanocrystals. Appl Phys Lett 2009; 94 : 211903.

6 Dohnalová K, Ondič L, Kůsová K, Pelant I, Rehspringer JL et al. White-emitting oxidized silicon nanocrystals: Discontinuity in spectral development with reducing size. J Appl Phys 2010; 107: 053102.

7 Vasiliev I, Chelikowsky JR, Martin RM. Surface oxidation effects on the optical properties of silicon nanocrystals. Phys Rev B 2002; 65: 121302.

8 Puzder A, Williamson AJ, Grossman JC, Galli G. Surface control of optical properties in silicon nanoclusters. J Chem Phys 2002; 117: 6721-6729.

9 Tsybeskov L, Vandyshev JV, Fauchet PM. Blue emission in porous silicon: oxygenrelated photoluminescence. Phys Rev B 1994; 49: 7821-7824.

10 Kanemitsu Y. Luminescence properties of nanometer-sized Si crystallites: core and surface states. Phys Rev B 1994; 49: 16845

11 Dohnalová K, Žídek K, Ondič L, Kůsová K, Cibulka $O$ et al. Optical gain at the F-band of oxidized silicon nanocrystals. J Phys D Appl Phys 2009; 42: 135102.

12 Luterová K, Pelant I, Mikulskas I, Tomasiunas R, Muller D et al. Stimulated emission in blue-emitting $\mathrm{Si}^{+}$-implanted $\mathrm{SiO}_{2}$ films? J App/ Phys 2002; 91: 2896-2900.

13 Valenta J, Fučíková A, Pelant I, Kůsová K, Dohnalová K et al. On the origin of the fast photoluminescence band in small silicon nanoparticles. New J Phys 2008; 10: 073022.

14 Belomoin G, Therrien J, Nayfeh M. Oxide and hydrogen capped ultrasmall blue luminescent Si nanoparticles. Appl Phys Lett 2000; 77: 779-781.

15 Cazzanelli M, Kovalev D, Dal Negro L, Gaburro Z, Pavesi L. Polarized optical gain and polarization-narrowing of heavily oxidized porous silicon. Phys Rev Lett 2004; 93. 207402.

16 Poddubny AN, Dohnalová K. Direct band gap silicon quantum dots achieved via electronegative capping. Phys Rev B 2014; 90: 245439.

17 Dohnalová K, Poddubny AN, Prokofiev AA, de Boer WDAM, Umesh CP et al. Surface brightens up Si quantum dots: direct bandgap-like size-tunable emission. Light Sci Appl 2013; 2: e47.

18 Kůsová K, Hapala P, Valenta J, Jelínek $\mathrm{P}$, Cibulka $\mathrm{O}$ et al. Direct bandgap silicon: tensile-strained silicon nanocrystals. Adv Mater Inter 2014; 1: 1300042.

19 Zyubin AS, Mebel AM, Lin SH, Glinka YD. Photoluminescence of silanone and dioxasilyrane groups in silicon oxides: A theoretical study. J Chem Phys 2002; 116: 9889-9896.

20 Salh R. Defect related luminescence in silicon dioxide network: a review. INTECH Open Access Publisher. (Croatia, EU), 2011. 
21 Fitting HJ, Barfels T, Trukhin AN, Schmidt B, Gulans A et al. Cathodoluminescence of $\mathrm{Ge}^{+}, \mathrm{Si}^{+}$, and $\mathrm{O}^{+}$implanted $\mathrm{SiO}_{2}$ layers and the role of mobile oxygen in defect transformations. J Non-Cryst Solids 2002; 303: 218-231.

22 Uchino T, Kurumoto N, Sagawa N. Structure and formation mechanism of blue-lightemitting centers in silicon and silica-based nanostructured materials. Phys Rev $B 2006$; 73: 233203.

23 Vaccaro L, Morana A, Radzig V, Cannas M. Bright visible luminescence in silica nanoparticles. J Phys Chem C 2011; 115: 19476-19481.

24 Carbonaro CM, Corpino R, Ricci PC, Chiriu D. Sensitivity to oxygen environments of mesoporous silica samples with different porosities. J Non-Cryst Solids 2014; 401: 60-65.

25 Glinka YD, Lin SH, Chen YT. Two-photon-excited luminescence and defect formation in $\mathrm{SiO}_{2}$ nanoparticles induced by 6.4-eV ArF laser light. Phys Rev B 2000; 62: 4733-4743.

26 Anjiki A, Uchino T. Visible photoluminescence from photoinduced molecular species in nanometer-sized oxides: crystalline $\mathrm{Al}_{2} \mathrm{O}_{3}$ and amorphous $\mathrm{SiO}_{2}$ nanoparticles. J Phys Chem C 2012; 116: 15747-15755.

27 Glinka YD, Lin SH, Chen YT. The photoluminescence from hydrogen-related species in composites of $\mathrm{SiO}_{2}$ nanoparticles. Appl Phys Let 1999; 75: 778-780.

28 Tamura H, Rückschloss M, Wirschem T, Vepřek S. Origin of the green/blue luminescence from nanocrystalline silicon. Appl Phys Lett 1994; 65: 1537-1739.

29 Loni A, Simons AJ, Calcot PDJ, Canham LT. Blue photoluminescence from rapid thermally oxidized porous silicon following storage in ambient air. J App/ Phys 1995; 77: 3557-3559.

30 Skuja L. The origin of the intrinsic $1.9 \mathrm{eV}$ luminescence band in glassy $\mathrm{SiO}_{2}$. J NonCryst Solids 1994; 179: 51-69.

31 Vaccaro L, Cannas M, Radzig V, Boscaino R. Luminescence of the surface nonbridging oxygen hole center in silica: Spectral and decay properties. Phys Rev B 2008; 78: 075421.

32 Vaccaro L, Cannas M, Boizot B, Parlato A. Radiation induced generation of non-bridging oxygen hole center in silica: Intrinsic and extrinsic processes. J Non-Cryst Solids 2007; 353: 586-589.

33 Munekuni S, Yamanaka T, Shimogaichi Y, Tohmon R, Ohki Y et al. Various types of nonbridging oxygen hole center in high-purity silica glass. J Appl Phys 1990; 68: 1212-1217.

34 Skuja L, Kajihara K, Hirano M, Hosono H. Oxygen-excess-related point defects in glassy/ amorphous $\mathrm{SiO}_{2}$ and related materials. Nucl Instrum Methods Phys Res B 2012; 286: 159-168.

35 Skuja L. Optically active oxygen-deficiency-related centers in amorphous silicon dioxide. J Non-Cryst Solids 1998; 239: 16-48.
36 Davies GL, McCarthy JE, Rakovich A, Gun'ko YK. Towards white luminophores: developing luminescent silica on the nanoscale. J Mater Chem 2012; 22: 7358-7365.

37 Spallino L, Vaccaro L, Sciortino L, Agnello S, Buscarino G et al. Visible-ultraviolet vibronic emission of silica nanoparticles. Phys Chem Chem Phys 2014; 16: 22028-22034.

38 Ricci PC, Casula R, Gulleri G, Fumagalli F, Carbonaro CM et al. Mechanical stress in silicon nanosized architectures: Defects of SOD processed silica filler. J Alloys Comp 2014; 602: 157-162.

39 Ricci PC, Gulleri G, Fumagalli F, Carbonaro M, Corpino R. Optical characterization of polysilazane based silica thin films on silicon substrates. App/ Surf Sci 2013; 265: 470-474.

40 Dohnalová K, Fučíková A, Umesh CP, Humpolíčková J, Paulusse JMJ et al. Microscopic origin of the fast blue-green luminescence of chemically synthesized non-oxidized silicon quantum dots. Small 2012; 8: 3185-3191.

41 Doğan I, Kramer NJ, Westermann RHJ, Dohnalová K, Smets AHM et al. Ultrahigh throughput plasma processing of free standing silicon nanocrystals with lognormal size distribution. J Appl Phys 2013; 113: 134306.

42 Bruhn B, Sangghaleh F, Linnros J. Fabricating single silicon quantum rods for repeatable single dot photoluminescence measurements. Phys Status Solidi A 2011; 208: 631-634.

43 Coenen T, Vesseur EJR, Polman A, Koenderink AF. Directional emission from plasmonic yagi-uda antennas probed by angle-resolved cathodoluminescence spectroscopy. Nano Lett 2011; 11: 3779-3784.

44 Brenny BJM, Coenen T, Polman A. Quantifying coherent and incoherent cathodoluminescence in semiconductors and metals. J Appl Phys 2014; 115: 244307.

45 Pacchioni G, Skuja L, Griscom DL. Defects in SiO2 and Related Dielectrics: Science and Technology. The Netherlands: Springer. 2000, p 73.

(c) (i) $(-)$ This work is licensed under a Creative Commons Attributionco NC ND NonCommercial-NoDerivs 4.0 International License. The images or other third party material in this article are included in the article's Creative Commons license, unless indicated otherwise in the credit line; if the material is not included under the Creative Commons license, users will need to obtain permission from the license holder to reproduce the material. To view a copy of this license, visit http:// creativecommons.org/licenses/by-nc-nd/4.0/

(C) The Author(s) 2017

Supplementary Information for this article can be found on the Light: Science \& Applications' website (http://www.nature.com/lsa). 